\title{
Molecular characterization of hepatitis $C$ virus in end-stage renal disease patients under hemodialysis
}

\author{
Rafael Alves da Silva ${ }^{1,2}$ (iD $\mid$ Jardelina de Souza Todão ${ }^{1}$ | \\ Fernando Luiz Kamitani $^{2}$ | Antonio Eduardo Benedito Silva ${ }^{1,3}$ \\ Roberto José de Carvalho-Filho ${ }^{1,3}$ | Maria Lucia Cardoso Gomes Ferraz ${ }^{1,3}$ \\ Isabel Maria Vicente Guedes de Carvalho ${ }^{1,2}$
}

\author{
${ }^{1}$ Laboratório de Hepatologia Molecular \\ Aplicada (LHeMA), Departamento de \\ Gastroenterologia, Unidade de Hepatologia, \\ Universidade Federal de São Paulo, UNIFESP, \\ São Paulo, Brazil \\ 2 Instituto Butantan, Laboratório de \\ Parasitologia, São Paulo, Brazil \\ 3 Departamento de Gastroenterologia, \\ Unidade de Hepatologia, Universidade Federal \\ de São Paulo, UNIFESP, São Paulo, Brazil \\ Correspondence \\ Rafael Alves da Silva, Instituto Butantan, \\ Laboratório de Parasitologia, Av. Vital Brasil \\ $n^{\circ} 1500$, Butantã, São Paulo, Brazil, CEP \\ 05503-900. \\ Email: alvesr.bio@gmail.com

\section{Funding information} \\ Coordenação de Aperfeiçoamento de Pessoal \\ de Nível Superior, Grant number: \\ 88881.132760/2016-01; Fundação de \\ Amparo à Pesquisa do Estado de São Paulo, \\ Grant number: 2012/18168-2
}

New direct-acting antiviral (DAA) agents are in development or already approved for the treatment of chronic hepatitis $C$ virus (HCV) infection. The effectiveness of these drugs is related to the previous existence of resistant variants. Certain clinical conditions can allow changes in immunological characteristics of the host and even modify genetic features of viral populations. The aim of this study was to perform HCV molecular characterization from samples of end-stage renal disease patients on hemodialysis (ESRD-HD). Nested PCR and Sanger sequencing were used to obtain genetic information from the NS5B partial region of a cohort composed by 86 treatment-naïve patients. Genomic sequences from the Los Alamos databank were employed for comparative analysis. Bioinformatics methodologies such as phylogenetic reconstructions, informational entropy, and mutation analysis were used to analyze datasets separated by geographical location, HCV genotype, and renal function status. ESRD-HD patients presented HCV genotypes $1 \mathrm{a}(n=18), 1 \mathrm{~b}(n=16), 2 \mathrm{a}(n=2)$, $2 \mathrm{~b}(n=2)$, and $3 \mathrm{a}(n=4)$. Control subjects were infected with genotypes $1 \mathrm{a}(n=11), 1 \mathrm{~b}$ $(n=21), 2 \mathrm{~b}(n=4)$, and $3 \mathrm{a}(n=8)$. Dataset phylogenetic reconstruction separated HCV subtype $1 a$ into two distinct clades. The entropy analysis from the ESRD-HD group revealed two amino acid positions related to an epitope for cytotoxic $T$ lymphocytes and T helper cells. Genotype 1a was found to be more diverse than subtype $1 \mathrm{~b}$. Also, genotype 1a ERSD-HD patients had a higher mean of amino acids changes in comparison to control group patients. The identification of specific mutations on epitopes and high genetic diversity within the NS5B HCV partial protein in hemodialysis patients can relate to host immunological features and geographical distribution patterns. This genetic diversity can affect directly the new DAA's resistance mechanisms.

KEYWORDS

evolution, genetic variability, genetics, hepatitis $C$ virus, mutation, virus classification 


\section{1 | INTRODUCTION}

Patients with end-stage renal disease (ESRD) under hemodialysis (HD) are exposed to an increased risk of acquiring pathogens infections through nosocomial ways, including viral hepatitis infections. ${ }^{1,2}$ The occurrence of hepatitis $\mathrm{C}$ virus (HCV) in HD patients is variable among different countries and medical centers, mostly associated with medical assistance quality. ${ }^{3}$ Nosocomial infection is a larger problem when dealing with disease without a vaccine, and recent researches indicate that this way of transmission is still a great risk for HCV infection and outbreaks inside hemodialysis units. ${ }^{4-7}$ Patients with ESRD infected with HCV are associated with a greater morbidity and mortality. ${ }^{8}$ The kidney function in the ESRD population plays a crucial role in the HCV treatment pharmacokinetics. ${ }^{9}$ Ribavirin-induced anemia is caused by the accumulation in erythrocytes subsequent to active unidirectional transmembrane transport. ${ }^{10}$ Patients with kidney disease tend to have baseline anemia, turning the conventional treatment hard to manage on chronic renal patients. ESRD-HD patients also present platelet function abnormalities, lower aminotransferase activity, and lower humoral response as factors that can aggravate HCV prognosis. ${ }^{11}$

The viral polymerase lacks of proofreading function and high replication rate contribute to a high genetic diversity even between variants from the same viral subtype. For $\mathrm{HCV}$, the estimated mutation rate in vivo was placed as $1.15 \pm 0.2 \times 10^{-4}$ substitutions per site replicated, ${ }^{12,13}$ creating a viral complexity genetic information on a host called quasispecies. ${ }^{14}$ Different factors can prevent the existence of this high diversity on the host, such as immunological pressures, good replication fitness by the virus to keep infecting new cells, and the treatment that can cause an adaptive effect on the viral population.

$\mathrm{HCV}$ populations of high genetic diversity can be found on chronic patients with normal renal function and presenting no immunological disorders. ${ }^{14-16}$ High genetic diversity is seen in phylogeographic patterns, genotype separation, and diverse subtypes. ${ }^{17}$ The absence of selective pressure caused by the lack of immunological function can also lead to a higher mutation fixation rate, representing a higher complexity on the HCV quasispecies composition in ESRD-HD patients. ${ }^{18}$

With new direct-acting antiviral (DAA) drugs for HCV infection treatment, resistance issues became easier to cope with.

Resistance-associated variants (RAV) searches for protease, polymerase, and NS5A inhibitors from sequence databases ${ }^{19-21}$ and untreated patients from different locations ${ }^{22-28}$ have already been performed. The presence of lower genetic barriers on specific subtypes is caused by the fixation of synonymous mutations on resistance-associated codons ${ }^{19}$ and the recent communication of long-term persistence of NS5A drug resistance mutations after the end of treatment ${ }^{29}$ raise important questions about new treatment strategies.

Data associating viral treatment resistance to genetic diversity from intra-host populations and the intrinsic difficulties to treat renal disease patients raise questions about the real genetic diversity from that special population.
The aim of this study was to characterize the genetic diversity and resistance mutation frequency of the ERSD-HD patients from the gastroenterology department of the Universidade Federal de São Paulo in relation to chronic-infected patients with normal renal function.

\section{2 | MATERIALS AND METHODS}

Plasma samples of $86 \mathrm{HCV}$ chronically infected patients were drafted at gastroenterology department of Universidade Federal de São Paulo (UNIFESP; São Paulo, Brazil). Patients were separated into two groups. The control group composed of subjects with normal renal function, chronical HCV infection, and the absence of coinfection. The HD group was formed by HCV chronically infected patients undergoing hemodialysis treatment sessions for at least 1 year. The 1 -year period of HD was chosen to able the viral population to establish a genetically diverse population according to the host-blunted immunological profile. Written informed consent was obtained from each patients and the study protocol was approved by the hospital ethics board in accordance with the 1975 Declaration of Helsinki. ${ }^{30}$

HCV RNA extractions were performed using the QIAamp Viral RNA Kit (Qiagen [Uniscience do Brasil], Sao Paolo, Brazil) starting with $140 \mu \mathrm{L}$ of serum, according to the manufacturer's recommendations and cDNA was produced using reverse transcriptase (Invitrogen) with random primers.

A two-step (nested) PCR protocol was used for the NS5B gene primer set (NS5B_243/NS5B_242 for the first PCR reaction and NS5B_Pr3/NS5B_Pr5 for the second reaction). ${ }^{31,32}$ The fragment amplified from the NS5B polymerase has already been tested for phylogenetic signal intensity. ${ }^{33}$ PCR products were purified using Illustra GFX PCR Purification Kit (GE Healthcare, Buckinghamshire, UK).

Capillary electrophoresis sequencing was performed with $A B I$ PRISM BigDye Terminator Cycle Sequencing Ready Reaction Kit (Life Technologies, Carlsbad, CA) on an ABI PRISM 3500 automatic sequencer (Life Technologies) according to the manufacturer's instructions. All the sequences were submitted to GenBank under the accessions numbers KP057692-KP057795.

After sequencing, contigs were created using Phred/Phrap/ Consed. $^{34,35}$ For accurate genotyping, the samples contigs and 14 reference sequences (AF009606_1a, D90208_1b, AY05129_1c, D14853_1c, D00944_2a, D10988_2b, AB047639_2a, D50409_2c, D17763_3a, D49374_3b, D28917_3a, Y11604_4a, Y13184_5a, Y12083_6a, D84262_6b e EF108306_7a) were aligned using Clustal $X 2.0{ }^{36}$ The best substitution model was identified using JModelTest $2 .{ }^{37}$ A phylogenetic tree was estimated from a HKY DNA substitution mode $^{38}$ with 1000 bootstrap pseudo replicates ${ }^{39}$ using Seaview 4.0 and PHYML package with Neighbor Joining method. ${ }^{40}$

A total of 82 Brazilian sequences belonging to subtype $1 \mathrm{a}$ and 161 sequences of subtype $1 \mathrm{~b}$ were obtained with the in silico search of Brazilian HCV polymerase fragment sequences on Los Alamos HCV database (https://hcv.lanl.gov/content/index) ${ }^{41}$ and Genbank (https:// www.ncbi.nlm.nih.gov/genbank/). Datasets of subtypes $1 \mathrm{a}$ and $1 \mathrm{~b}$ with in silico and target sequences obtained on that work were used to obtain 
a more detailed genetic reconstruction analysis with local geographical information. Both datasets demonstrated a good phylogenetic signal for the fragment. Phylogenetic reconstruction by maximum likelihood was made with both subtypes with a $\mathrm{HKY}+\mathrm{I}+\mathrm{G}$ model ${ }^{38}$ as indicated by the software jModeltest 2.0. ${ }^{37}$ Clade patterns among sequences from genetics databases, control patients, and HD patients were analyzed.

The genetic diversity of both groups was obtained comparing the number of nucleotide and amino acids alterations on datasets separated by genotype according to the reference sequence clade formation pattern. The means of nucleotide and amino acid divergence between sequences groups were tested for normality and then compared with Student's $t$-test for no related samples with SPSS software. ${ }^{42} P$ values lower than 0.05 were used to determine whether genetic changes between genotypes and analysis groups were significantly different.

\section{3 | RESULTS}

Genetic sequences from 86 plasma samples were obtained after Phred/ Phrap screening and contig assembly, 42 patients with ESRD (HD group) and 44 patients with normal renal function (control group). Neighbor joining phylogenetic reconstruction was performed with 16 reference sequences to generate genotype and subtype for each sample. All the clades that characterized genotypes and subtypes separation had Bootstrap value greater than 70 (Suplemmentary Figure 1).

HD group patients presented HCV genotypes $1 \mathrm{a}(n=18), 1 \mathrm{~b}$ $(n=16), 2 a(n=2), 2 b(n=2)$, and $3 a(n=4)$. Control subjects were infected with genotypes $1 \mathrm{a}(n=11), 1 \mathrm{~b}(n=21), 2 \mathrm{~b}(n=4)$, and $3 \mathrm{a}(n=8)$ (Table 1).

The 1a subtype dataset phylogenetic reconstruction demonstrated 16 sequences from this work with a common ancestral origin (eight monophyletic clades). Of the eight monophyletic clades, seven of them were made of sequences from the same analysis group (six clades formed with HD group sequences and one clade with control group sequences). Only one of the eight monophyletic clades is formed by sequences from different analysis group (HD and control group). Thirteen sequences from our 1a patient's sequences formed clades with local databank genetic sequences or as clades outgroups.

Subtype 1b phylogenetic reconstruction presented only two clades with monophyletic origin between the two groups analyzed.

TABLE 1 Genotype distribution between the HD and control patients groups

\begin{tabular}{|llll}
\hline Genotype & HD Group & Control & Total \\
\hline $1 \mathrm{a}$ & 18 & 11 & 29 \\
\hline $1 \mathrm{~b}$ & 16 & 21 & 37 \\
\hline $2 \mathrm{a}$ & 2 & - & 2 \\
\hline $2 \mathrm{~b}$ & 2 & 4 & 6 \\
\hline $3 a$ & 4 & 8 & 12 \\
\hline Total & 42 & 44 & 86 \\
\hline
\end{tabular}

One of the clades is composed of two HD group patients and another clade is composed of two sequences from different analysis group. Genotype $1 \mathrm{~b}$ remaining sequences $(n=33)$ formed clades with genetic database sequences (Figure 1).

In both genotypes, none of HD group patients who formed monophyletic clades were from the same dialysis center.

Informational entropy analysis was realized on the NS5B polymerase fragment of subtypes $1 \mathrm{a}$ and $1 \mathrm{~b}$ separated by patient's groups and database sequences. The search aimed for amino acid positions with higher entropy values only present on HD group HCV sequences, not on control and genetic database sequences. Genotype 1a group presented two positions with entropy peaks higher than 0.2 bits ( 23 and 26 of the fragment, 2668 and 2671 from $\mathrm{H} 77$ sequence reference). Subtype $1 \mathrm{~b}$ sequences presented a similar profile with one entropy peak position (25 from the fragment, 2670 from H77 reference) only on HD group. A second position close to the first presented an entropy peak only on group HD and database sequences (27 from the fragment, 2672 from $\mathrm{H} 77$ reference) absent on Brazilian sequences (Figure 2).

All four positions are closely related to a particular epitope for cytotoxic T lymphocytes and T helper cells recognition (CCDLDPQARVAl; positions 2662-2673 in the H77 reference sequence) (Figure 2).

To assess genetic diversity, the mean of amino acid and nucleotide changes using the subtype reference sequence was made (AF009606 for 1a subtype and D90208 for $1 \mathrm{~b}$ ). The mean changes were significantly higher $(P$ value $<0.05)$ on subtype $1 \mathrm{~b}$ than subtype $1 \mathrm{a}$ in both the nucleotides $(12.75 \pm 3.4$ vs. $30.42 \pm 3.7 ; P>0.001)$ and amino acids changes ( $2.25 \pm 1.2$ vs. $6.67 \pm 0.9 ; P>0.001)$ (Figure 3 ).

After that analysis, the mean of amino acid and nucleotide changes using the subtype reference sequence as a model for the count was made on datasets separated for subtypes and using both analyses groups (Figure 4). Nucleotide count changes were significantly higher for HD group on subtype $1 \mathrm{a}(14.53 \pm 2.4$ vs. $10 \pm 2.8 ; P<0.0001)$ and subtype $1 b$ ( $32.50 \pm 1.7$ vs. $28.89 \pm 4.0 ; P<0.004)$. Amino acid changes were only significant on subtype 1 a (1a: $2.53 \pm 1.2$ vs. $1.82 \pm 1.0$; $P<0.001 / 1$ b: $7 \pm 0.96$ vs. $6.4 \pm 0.9$ ).

\section{4 | DISCUSSION}

Since the start of INF and RBV treatment, different factors influenced how resistance is treated. HCV quasispecies genetic diversity studies are focused on proteins as NS5A and envelope proteins (E1 and E2), usually associated with INF resistance. ${ }^{43,44}$ Evidence of high quasispecies diversity on pretreatment populations has already been related to the poor response to INF and Ribavirin treatment, although the lack of direct association with resistance to treatment and genetic mechanisms. ${ }^{45-48}$ The genotype distribution on our samples was in accordance with another works that place genotype 1 as the most present in Brazil and the presence of genotypes 2 and 3 on a lower frequency. ${ }^{49,50}$

$\mathrm{HCV}$ genotype distribution on HD patients can be related to specific event transmissions on medical units. ${ }^{51}$ Our data demonstrated a 


\section{Genotype 1a}

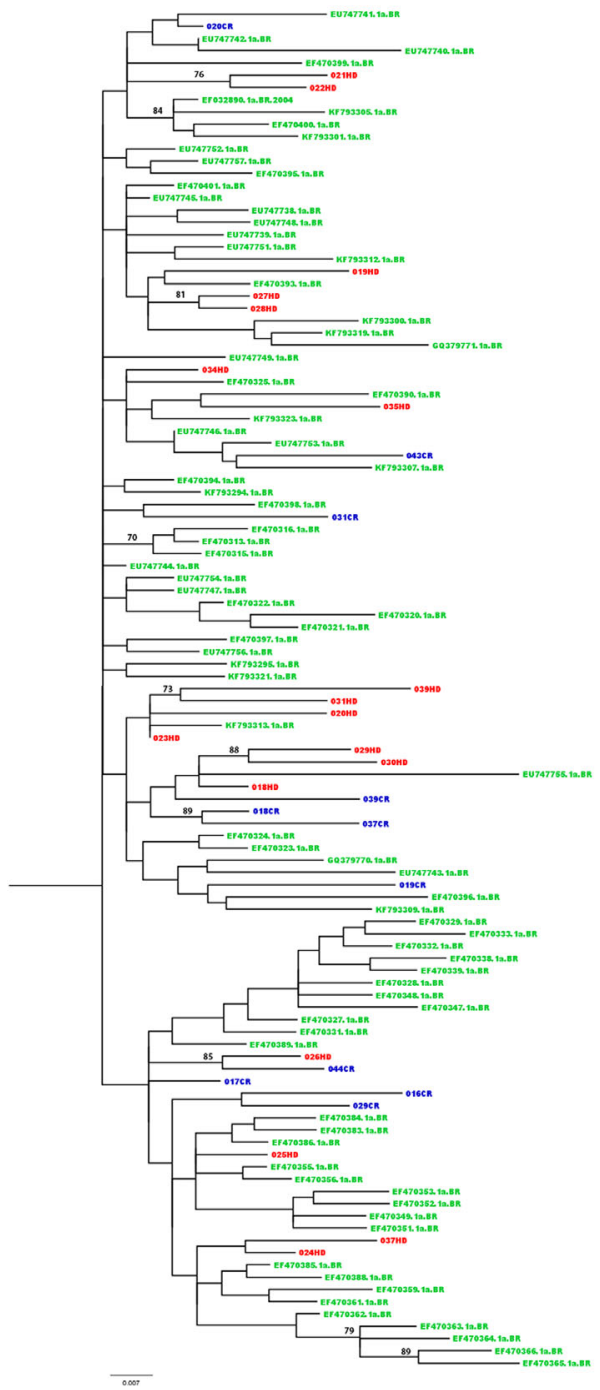

\section{Genotype 1b}

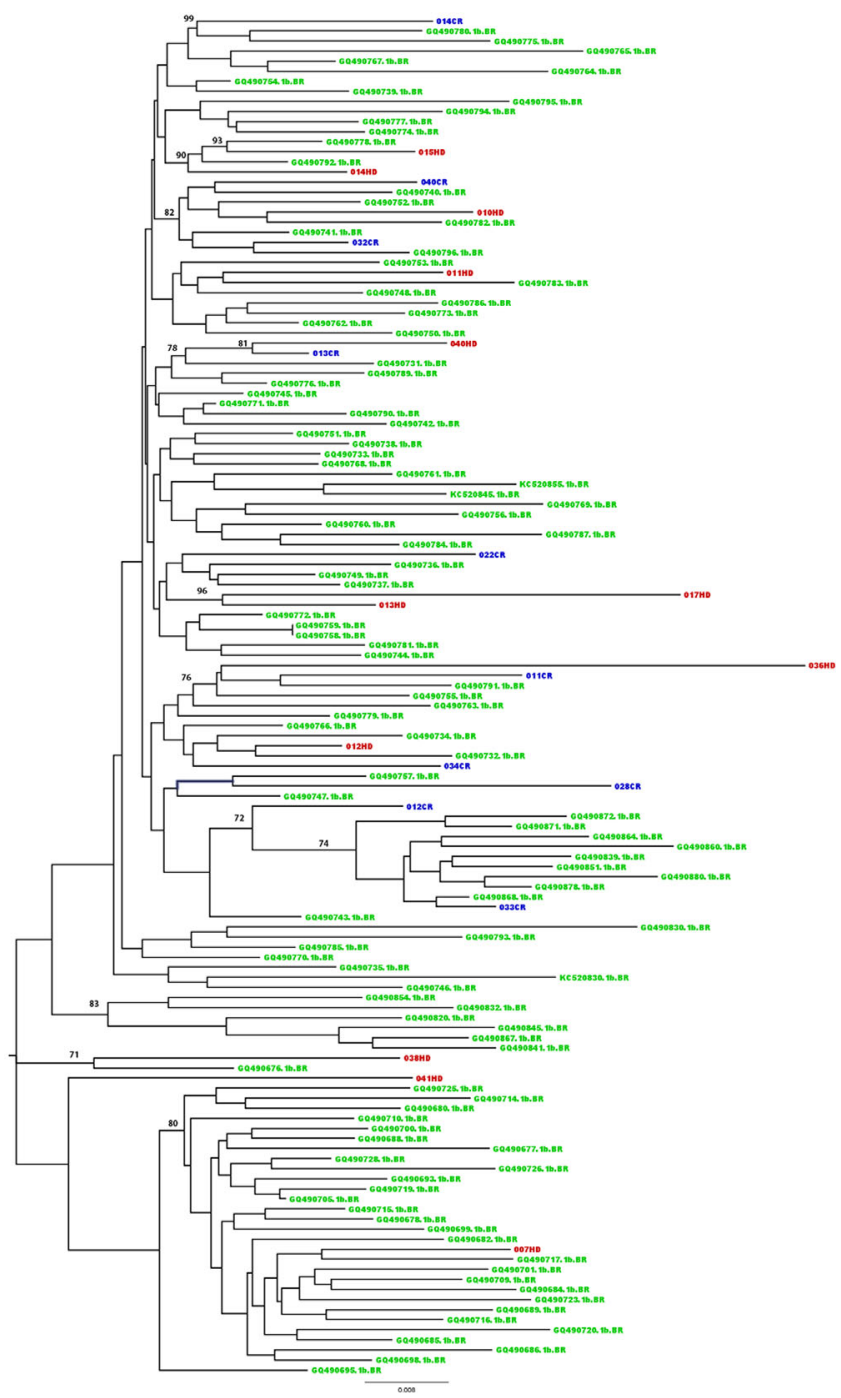

FIGURE 1 Phylogenetic reconstruction for both subtypes $1 \mathrm{a}$ and $1 \mathrm{~b}$ made by maximum likelihood method and $\mathrm{HKY}+\mathrm{G}+\mathrm{I}$ evolutionary model. Subtype 1a analysis employed 111 sequences and subtype 1b, 198. Patients from control group are in blue, patients from hemodialysis group on red, and Brazilian database sequences on green. Bootstrap percentage are on the respective node

homogeneity when considering subtypes distribution between groups analyzed, what can be related to the small number of patients in both groups and subtypes. Unlike hepatitis B genotype distribution between hemodialysis patients that present the genotype $D$ as the mostly associated with that particular group, ${ }^{1,52}$ our group of patients demonstrated a similar genotype distribution with normal renal function and ESRD patients.

Quasispecies diversity is an adaptive mechanism characterized by the virus capacity to escape from immunological host pressures also considered on the new HCV DAA treatments and the acquisition of specific resistance mutations. Resistance associated variants are seen on minor viral populations in the patient that rise along the treatment. During the treatment, the resistance-associated populations enhance its frequency thought lack of competition, even with lower replication fitness. ${ }^{53}$ One of the differences between subtypes observed in this work is the higher genetic diversity demonstrated as mean of changes on amino acid and nucleotide differences. Romano and Carvalho-Mello ${ }^{54}$ proposed that phylodynamics differences can be explained by the older origin of subtype $1 \mathrm{~b}$. If so, a saturation rate in the mutations could make difficult to visualize genetic patterns of changes on different groups. Our analysis demonstrated a clear and significant higher mean of changes on subtype 1a samples.

$\mathrm{HCV}$ intrinsic capacity to create a high genetic diversity contributes to several adaptive mutations that can lead to a escape from host's immune system. One of the features caused by the high genetic diversity is amino acid changes in epitopes. Studies already demonstrated virus neutralizing antibody response as an important viral evolution variable. ${ }^{55}$ Modifications on epitopes regions has been employed by the virus as a way to blunt regulation of $\mathrm{T}$ cell response and avoid early virological clearance, although $20 \%$ of patients have 


\section{Genotype 1a}
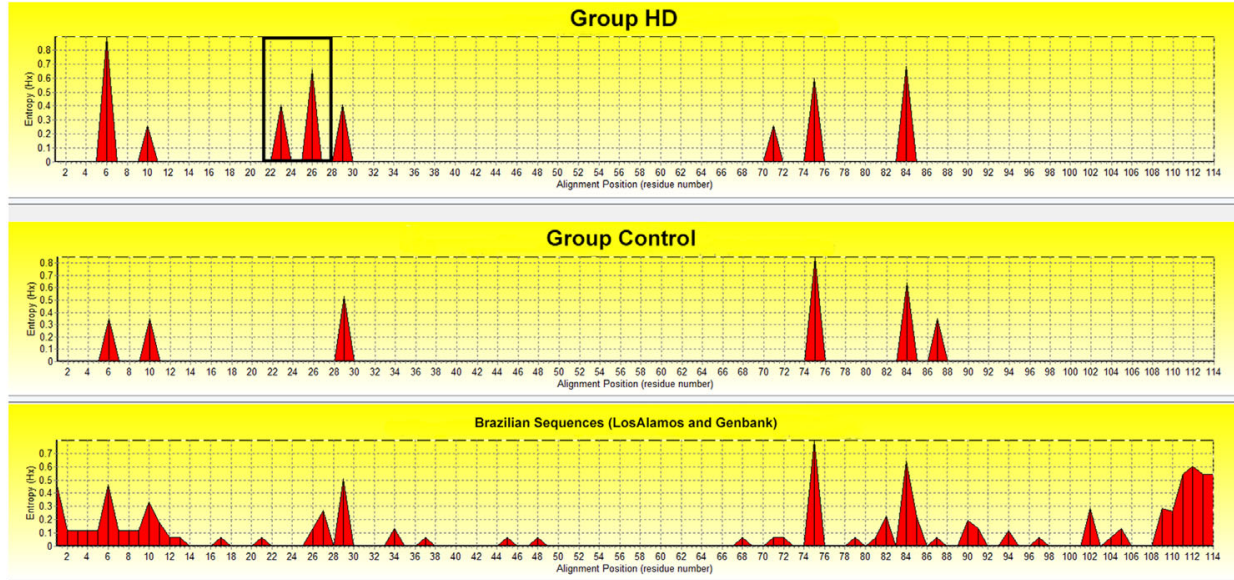

\section{Genotype 1b}
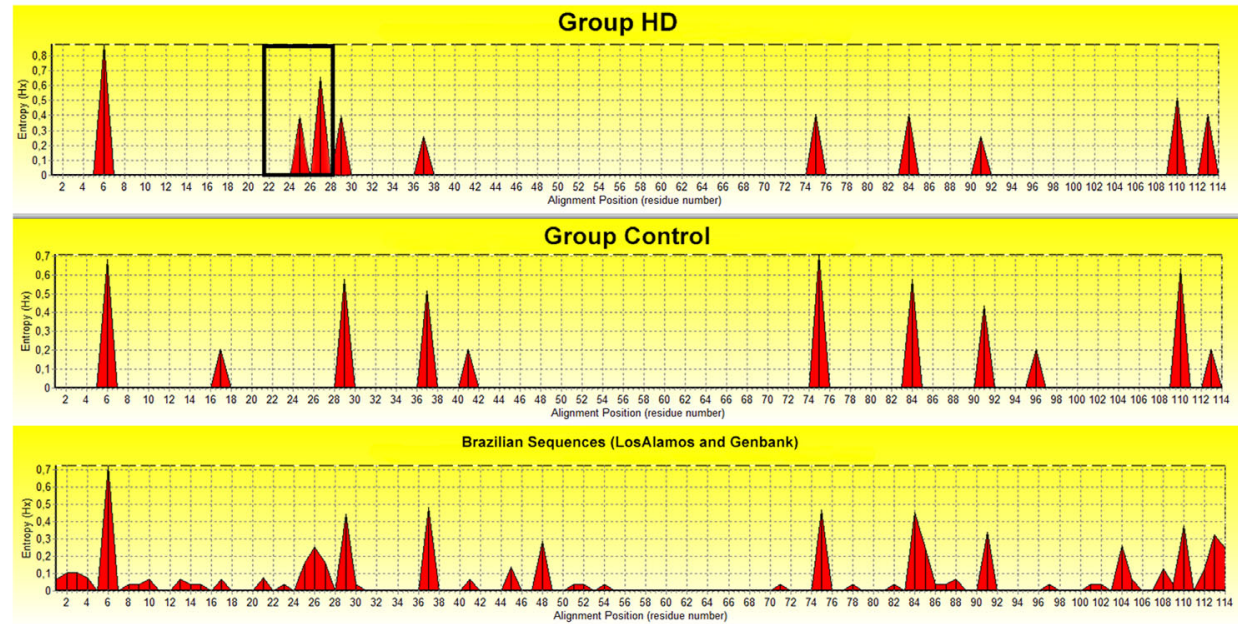

FIGURE 2 Informational Shannon entropy graphs for all sequences from genotype 1 obtained in this work for HD, control, and database Brazilian sequences groups. The entropy is represented in bits for each residue numbers in amino acids positions from the fragment analyzed

early virological clearance and are protected from reinfection indicating the possibility of a vaccine development. ${ }^{56-58}$ It remains unclear how the evolution of epitopes regions on different host immune system pressures takes place.

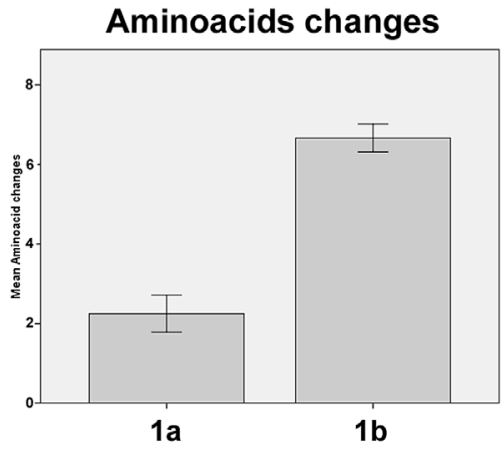

The Shannon informational entropy is a tool that can calculate the amount of informational complexity on homologue amino acids and nucleotides sequences. Epitope regions exhibit a tendency to have lower entropy values, consequently, a higher informational conservation.

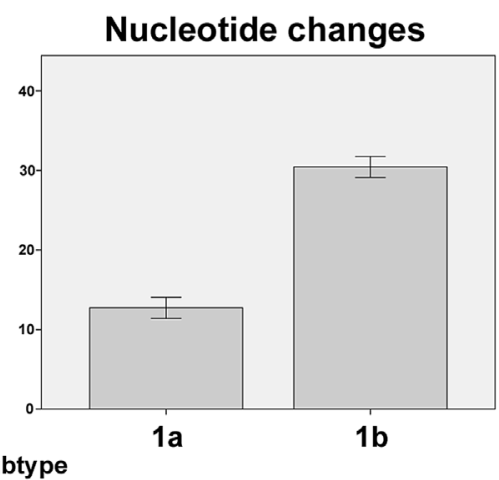

FIGURE 3 Graph of means of amino acids and nucleotide alterations from subtypes 1a and $1 \mathrm{~b}$ sequences of groups HD ( $n=31$ ) and control group $(n=30)$. The mean difference was statistically significant by the t-Student test for non-related samples with $P<0.0001$ 


\section{Aminoacid Changes Nucleotide Changes}

\section{Subtype 1a}
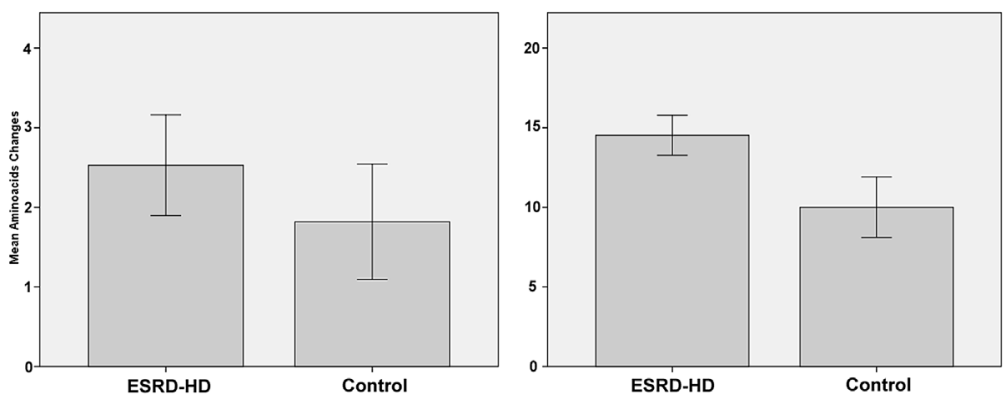

Subtype 1b
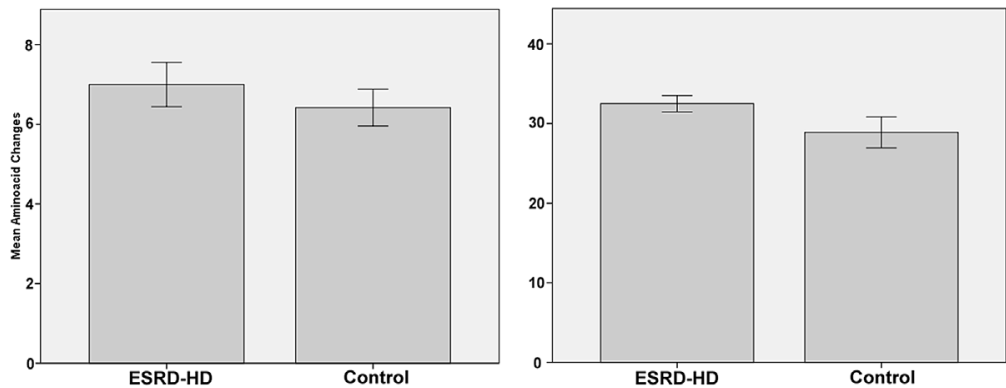

FIGURE 4 Means of amino acids and nucleotide changes from ESRD-HD group and control group for subtypes 1 a sequences $(n=28)$ and $1 \mathrm{~b}(n=33)$ from both groups. The mean difference was statistically significant in both genotypes on nucleotide changes by the $t-S t u d e n t$ test for non-related samples with $P<0.0001$ for the difference on subtype $1 \mathrm{a}$ and $P<0.004$ for subtype $1 \mathrm{~b}$

This can be explained by the necessity of protein structural stability for cell recognition. ${ }^{59}$ In our work, entropy peaks were found on two positions related to the NS5B recognition epitopes.

Patients under hemodialysis present several complications, including a blunted immunological system. ${ }^{18}$ The absence of some immunological pressure on the viral evolution can be the main reason to the nucleotide changes accumulation. The non-significant amino acids difference between ERSD-HD and control group can be related to the conformational protein conservation necessity to maintain its function. The increased nucleotide genetic diversity can be associated with factors as genetic memory by the presence of degenerates codons that are prone to resistance development or that can cause an easier escape from the immune system, making virions better fitted to high selective pressures situations. The hypothesis that part of the difficulties to obtain sustained virological response in hemodialysis patients in comparison to normal kidney function patients could be related to the larger capacity of the virus to acquire epitope escape mutations is sustained by these findings.

The RNA-dependent RNA-polymerase HCV protein is a highly functional protein that demands a certain level of conservation to maintain a high replication fitness, specifically on the active site. ${ }^{60}$ Necessity of high replication fitness through informational conservation is opposed to the challenge imposed by the immunological system. As a consequence of host's immunological pressure absence in ERSDHD patients, viral particles are free to accumulate mutations through the RNA molecule enhancing the chance to present nucleotide and amino acid changes on recognitions epitopes. Unless those mutations alter the replication fitness, the tendency to maintain polymorphic populations is increased. Admitting the hypothesis that genetic diverse populations have a more chance to escape antiviral treatment, ${ }^{61}$ patients who undergo hemodialysis are more likely to present that type of complexity viral population.

\section{5 | CONCLUSION}

Despite the clade formation pattern of HCV subtype 1a from the same hemodialysis patient analysis group, no relations about the hemodialysis centers were found, discarding nosocomial transmission between patients. The minor 1a subtype genetic diversity can be associated to the more recent origin, and less saturated mutation rate between isolates. ERSD-HD patients are known to have a blunted immunological system and the significant differences on nucleotide mutations seen on this work could be related to minor immunological pressures imposed by that specific population.

\section{ACKNOWLEDGMENTS}

This work was supported by Fundação de Amparo a Pesquisa do estado de São Paulo (FAPESP, grant number: 2012/18168-2), and Coordenação de Aperfeiçoamento de Pessoal de Nível Superior (CAPES PDSE: 88881.132760/2016-01). 


\section{ORCID}

Rafael Alves da Silva (iD http://orcid.org/0000-0003-3473-9412

\section{REFERENCES}

1. Rinonce $H T$, Yano $Y$, Utsumi T, et al. Hepatitis $B$ and $C$ virus infection among hemodialysis patients in Yogyakarta, Indonesia: prevalence and molecular evidence for nosocomial transmission. J Med Virol. 2013;85:1348-1361.

2. Su Y, Norris JL, Zang C, Peng Z, Wang N. Incidence of hepatitis C virus infection in patients on hemodialysis: a systematic review and metaanalysis. Hemodial Int. 2013;17:532-541.

3. Fissell RB, Bragg-Gresham JL, Woods JD, et al. Patterns of hepatitis C prevalence and seroconversion in hemodialysis units from three continents: the DOPPS. Kidney Int. 2004;65:2335-2342.

4. Lauer GM, Walker BD. Hepatitis C virus infection. N Engl J Med. 2001;345:41-52.

5. Szücs M, Dencs Á, Varga E, et al. An archived serum sample as a clue for identifying the primary source of a nosocomial hepatitis $C$ virus outbreak in a haemodialysis unit. Arch Virol. 2014;159:2207-2212.

6. da Silva NMO, Germano FN, Mendoza-Sassi RA, Seuánez HN, Soares MA, de Martinez AMB. Evidence of association between hepatitis $C$ virus genotype $2 \mathrm{~b}$ and nosocomial transmissions in hemodialysis centers from southern Brazil. Virol J. 2013;10:167.

7. Fabrizi F, Messa P. Transmission of hepatitis $C$ virus in dialysis units: a systematic review of reports on outbreaks. Int J Artif Organs. 2015;38:471-480.

8. Nakayama E, Akiba T, Marumo F, Sato C. Prognosis of anti-hepatitis C virus antibody-positive patients on regular hemodialysis therapy. J Am Soc Nephrol. 2000;11:1896-1902.

9. Al-Freah MAB, Zeino Z, Heneghan MA. Management of hepatitis $C$ in patients with chronic kidney disease. Curr Gastroenterol Rep. 2012;14:78-86.

10. Russmann S, Grattagliano I, Portincasa P, Palmieri VO, Palasciano G. Ribavirin-induced anemia: mechanisms, risk factors and related targets for future research. Curr Med Chem. 2006;13:3351-3357.

11. Fabrizi F. Hepatitis $C$ virus infection and dialysis: 2012 update. ISRN Nephrol. 2013;2013:159760.

12. Ribeiro RM, Li H, Wang S, et al. Quantifying the diversification of hepatitis c virus (HCV) during primary infection: estimates of the in vivo mutation rate. PLoS Pathog. 2012;8:e1002881.

13. Cuevas JM, González-Candelas F, Moya A, Sanjuán R. Effect of ribavirin on the mutation rate and spectrum of hepatitis $C$ virus in vivo. J Virol. 2009;83:5760-5764.

14. Domingo E, Gomez J. Quasispecies and its impact on viral hepatitis. Virus Res. 2007;127:131-150.

15. Simmonds P. Genetic diversity and evolution of hepatitis $C$ virus -15 years on. J Gen Virol. 2004;85:3173-3188.

16. Jardim ACG, Yamasaki LHT, de Queiróz ATL, et al. Quasispecies of hepatitis $C$ virus genotype 1 and treatment outcome with peginterferon and ribavirin. Infect Genet Evol. 2009;9:689-698.

17. Simmonds $\mathrm{P}$, Bukh J, Combet $\mathrm{C}$, et al. Consensus proposals for a unified system of nomenclature of hepatitis $C$ virus genotypes. Hepatology. 2005;42:962-973.

18. Kato $\mathrm{S}$, Chmielewski $\mathrm{M}$, Honda $\mathrm{H}$, et al. Aspects of immune dysfunction in end-stage renal disease. Clin J Am Soc Nephrol. 2008;3:1526-1533.

19. Cento V, Mirabelli C, Salpini R, et al. HCV genotypes are differently prone to the development of resistance to linear and macrocyclic protease inhibitors. PLOS ONE. 2012;7:e39652.

20. Alves R, Queiroz ATL, Pessoa MG, et al. The presence of resistance mutations to protease and polymerase inhibitors in hepatitis $C$ virus sequences from the Los Alamos databank. J Viral Hepat. 2013;20:414-421.

21. Nakamoto S, Kanda T, Wu S, Shirasawa H, Yokosuka O. Hepatitis C virus NS5A inhibitors and drug resistance mutations. World $J$ Gastroenterol. 2014;20:2902-2912.

22. Kuntzen T, Timm J, Berical A, et al. Naturally occurring dominant resistance mutations to hepatitis $\mathrm{C}$ virus protease and polymerase inhibitors in treatment-naïve patients. Hepatology. 2008;48:1769-1778.

23. Peres-da-Silva A, de Almeida AJ, Lampe E. Mutations in hepatitis $C$ virus NS3 protease domain associated with resistance to specific protease inhibitors in antiviral therapy naïve patients. Arch Virol. 2010;155:807-811.

24. Fonseca-Coronado S, Escobar-Gutiérrez A, Ruiz-Tovar K, et al. Specific detection of naturally occurring hepatitis $\mathrm{C}$ virus mutants with resistance to telaprevir and boceprevir (protease inhibitors) among treatment-naïve infected individuals. J Clin Microbiol. 2012;50:281-287.

25. de Carvalho IMVG, Alves R, de Souza PAV-M, et al. Protease inhibitor resistance mutations in untreated Brazilian patients infected with HCV: novel insights about targeted genotyping approaches. J Med Virol. 2014;86:1714-1721.

26. Maimone S, Tripodi G, Musolino C, Cacciola I, Pollicino T, Raimondo G. Lack of the NS5B S282T mutation in HCV isolates from liver tissue of naive patients with HCV genotype-1b infection. Antivir Ther. 2014;20:245-247.

27. Aissa Larousse J, Trimoulet $P$, Recordon-Pinson $P$, et al. Natural prevalence of hepatitis $C$ virus ( $\mathrm{HCV}$ ) variants resistant to protease and polymerase inhibitors in patients infected with HCV genotype 1 in Tunisia. J Med Virol. 2014;86:1350-1359.

28. Fridell RA, Qiu D, Wang C, Valera L, Gao M. Resistance analysis of the hepatitis C virus NS5A inhibitor BMS-790052 in an in vitro replicon system. Antimicrob Agents Chemother. 2010;54:3641-3650.

29. Yoshimi S, Imamura M, Murakami E, et al. Long term persistence of NS5A inhibitor-resistant hepatitis C virus in patients who failed daclatasvir and asunaprevir therapy. J Med Virol. 2015;87:1913-1920.

30. WMA Declaration of Helsinki. Ethical principles for medical research involving human subjects. The World Medical Association.

31. Sandres-Sauné K, Deny P, Pasquier C, Thibaut V, Duverlie G, Izopet J. Determining hepatitis $C$ genotype by analyzing the sequence of the NS5b region. J Virol Methods. 2003;109:187-193.

32. Enomoto N, Takada A, Nakao T, Date T. There are two major types of hepatitis $\mathrm{C}$ virus in Japan. Biochem Biophys Res Commun. 1990;170:1021-1025.

33. de Carvalho-Mello IMVG, Filho JEM, Gomes-Gouvêa MS, et al. Molecular evidence of horizontal transmission of hepatitis $C$ virus within couples. J Gen Virol. 2010;91:691-696.

34. Ewing B, Hillier L, Wendl MC, Green P. Base-calling of automated sequencer traces using Phred. I. accuracy assessment. Genome Res. 1998;8:175-185.

35. Ewing B, Green P. Base-calling of automated sequencer traces using phred. II. Error probabilities. Genome Res. 1998;8:186-194.

36. Larkin MA, Blackshields G, Brown NP, et al. Clustal W and clustal $x$ version 2.0. Bioinformatics. 2007;23:2947-2948.

37. Darriba D, Taboada GL, Doallo R, Posada D. JModelTest 2: more models, new heuristics and parallel computing. Nat Methods. 2012;9:772.

38. Hasegawa M, Kishino H, Yano T. Dating of the human-ape splitting by a molecular clock of mitochondrial DNA. J Mol Evol. 1985;22:160-174.

39. Efron B, Halloran E, Holmes S. Bootstrap confidence levels for phylogenetic trees. Proc Natl Acad Sci USA. 1996;93:7085-7090.

40. Gouy M, Guindon S, Gascuel O. SeaView version 4: a multiplatform graphical user interface for sequence alignment and phylogenetic tree building. Mol Biol Evol. 2009;27:221-224.

41. Hraber PT, Leach RW, Reilly LP, Thurmond J, Yusim K, Kuiken C. Los Alamos hepatitis $C$ virus sequence and human immunology databases: an expanding resource for antiviral research. Antivir Chem Chemother. 2007;18:113-123. 
42. Kim TK. T test as a parametric statistic. Korean J Anesthesiol. 2015;68:540-546.

43. ElHefnawi MM, Zada S, El-Azab IA. Prediction of prognostic biomarkers for interferon-based therapy to hepatitis $C$ virus patients: a meta-analysis of the NS5A protein in subtypes 1a, 1b, and 3a. Virol J. 2010;7:130.

44. Bolcic F, Laufer N, Torres C, Cassino L, Reynoso R, Quarleri J. Longitudinal analysis of the $5^{\prime} \mathrm{UTR}, \mathrm{E} 2-\mathrm{PePHD}$ and NS5A-PKRBD genomic regions of hepatitis $C$ virus genotype $1 \mathrm{a}$ in association with the response to peginterferon and ribavirin therapy in HIV-coinfected patients. Antiviral Res. 2012;95:72-81.

45. Jardim AC, Bittar C, Matos RP, et al. Analysis of HCV quasispecies dynamic under selective pressure of combined therapy. BMC Infect Dis. 2013;13:61.

46. Jardim ACG, Yamasaki LHT, de Queiróz ATL, et al. Quasispecies of hepatitis $C$ virus genotype 1 and treatment outcome with peginterferon and ribavirin. Infect Genet Evol. 2009;9:689-698.

47. Bittar C, Jardim ACG, Yamasaki LHT, et al. On hepatitis C virus evolution: the interaction between virus and host towards treatment outcome. PLoS ONE. 2013;8:e62393.

48. Akram $M$, Idrees $M$, Hussain $A$, et al. Characterization of hepatitis $C$ Virus genotype 3a hypervariable region 1 in patients achieved rapid virological response to alpha interferon and ribavirin combination therapy. Virol J. 2011;8:253.

49. Lampe E, Lewis-Ximenez L, Espírito-Santo MP, et al. Genetic diversity of HCV in Brazil. Antivir Ther. 2013;18:435-444.

50. Campiotto S, Pinho JRR, Carrilho FJ, et al. Geographic distribution of hepatitis C virus genotypes in Brazil. Brazilian J Med Biol Res. 2005;38:41-49.

51. Spada E, Abbate I, Sicurezza E, et al. Molecular epidemiology of a hepatitis $C$ virus outbreak in a hemodialysis unit in Italy. J Med Virol. 2008;80:261-267.

52. Niloofar N, Manochehr M, Reza S-ZA. A study on genotypes of hepatitis $B$ virus among hemodialysis patients in Khuzestan province. Jundishapur J Microbiol. 2011;4:2.

53. Welsch C. Genetic barrier and variant fitness in hepatitis $C$ as critical parameters for drug resistance development. Drug Discov Today Technol. 2014;11:19-25.

54. Romano CM, de Carvalho-Mello IMVG, Jamal LF, et al. Social networks shape the transmission dynamics of hepatitis C virus. PLoS ONE. 2010;5:e11170.
55. Pestka JM, Zeisel MB, Bläser E, et al. Rapid induction of virusneutralizing antibodies and viral clearance in a single-source outbreak of hepatitis C. Proc Natl Acad Sci USA. 2007;104: 6025-6030.

56. Dustin LB, Cashman SB, Laidlaw SM. Immune control and failure in HCV infection-tipping the balance. J Leukoc Biol. 2014;96:1-14.

57. Losikoff PT, Mishra S, Terry F, et al. HCV epitope, homologous to multiple human protein sequences, induces a regulatory $\mathrm{T}$ cell response in infected patients. J Hepatol. 2015;62:48-55.

58. Ziegler S, Skibbe K, Walker A, et al. Impact of sequence variation in a dominant HLA-A*02-restricted epitope in hepatitis $C$ virus on priming and cross-reactivity of CD8+ T cells. J Virol. 2014;88: 11080-11090.

59. Alekseeva L, Nekrasov A, Marchenko A, et al. Cryptic B-cell epitope identification through informational analysis of protein sequenses. Vaccine. 2007;25:2688-2697.

60. Lesburg CA, Cable MB, Ferrari E, Hong Z, Mannarino AF, Weber PC. Crystal structure of the RNA-dependent RNA polymerase from hepatitis C virus reveals a fully encircled active site. Nat Struct Biol. 1999;6:937-943.

61. El-Shamy A, Hotta $\mathrm{H}$. Impact of hepatitis $\mathrm{C}$ virus heterogeneity on interferon sensitivity: an overview. World J Gastroenterol. 2014;20: 7555-7569.

\section{SUPPORTING INFORMATION}

Additional Supporting Information may be found online in the supporting information tab for this article.

How to cite this article: Alves da Silva R, de Souza Todão J, Kamitani FL, et al. Molecular characterization of hepatitis $\mathrm{C}$ virus in end-stage renal disease patients under hemodialysis. J Med Virol. 2018;90:537-544. https://doi.org/10.1002/jmv.24976 